\title{
ĐÁNH GIÁ ĐỘ CHÍNH XÁC CỦA MÔ HÌNH QUASIGEOID QUỐC GIA KHỞI ĐẦU VIGAC 2017
}

\author{
HÀ MINH HOÀ(1), LƯO'NG THANH THẠCH(2) \\ (1) Viện Khoa học Đo đạc và Bản đồ \\ ${ }^{(2)}$ Trường Đại học Tài nguyên Môi trường Hà Nội
}

\section{Tóm tắt:}

Bài báo khoa học này đã giới thiệu các kết quả xây dựng mô hình quasigeoid quốc gia khởi đầu VIGAC2017 với độ chính xác trong hệ quy chiếu tọa độ không gian khởi đầu VN2000-3D. Các kết quả kiểm tra cho thấy độ tin cậy và độ chính xác cao của mô hình quasigeoid quốc gia khởi đầu VIGAC 2017.

\section{1. Đặt vấn đề}

Việc xây dựng hệ quy chiếu tọa độ không gian quốc gia (hệ tọa độ động) là một trong những nội dung quan trọng của Chiến lược Phát triển ngành Đo đạc và Bản đồ đến năm 2020 đã được phê duyệt trong Quyết định số 33/2008/QĐ-TTg ngày 27/02/2008 của Thủ tướng Chính phủ và tập trung trong mình các thành tựu hiện đại nhất của Trắc địa cao cấp trong giai đoạn hiện nay. Như đã trình bày trong [Hà Minh Hòa và nnk, 2012], mấu chốt của việc giải quyết nhiệm vụ nêu trên là xây dựng mô hình quasigeoid độ chính xác cao trên lãnh thổ quốc gia. Hệ quy chiếu tọa độ không gian quốc gia được xây dựng trong mối liên kết với ITRF phải đảm bảo để sai số tương đối của các tọa độ không gian nằm ở mức $1.10^{-9}$ [Augath $\mathrm{W}$., Ihde J., 2002]. Để đạt được tiêu chí trên, trong [Hà Minh Hòa, 2014a] đã chứng minh được rằng độ chính xác của mô hình quasigeoid quốc gia phải cao hơn $\pm 4 \mathrm{~cm}$. Khi ký hiệu $z^{\prime}{ }^{\prime}$ là độ cao chuẩn quốc gia, $\zeta$ là độ cao quasigeoid quốc gia, $H$ là độ cao trắc địa quốc gia của điểm trắc địa, việc xây dựng mô hình quasigeoid quốc gia độ chính xác cao phải đảm bảo để luôn nhận được quan hệ $H=H^{\gamma}+\zeta$.Trong thực tiễn thế giới, hệ tọa độ không gian địa tâm OS Net (Ordnance Survay Network) của nước Anh được xây dựng dựa trên mô hình geoid OSGM02 (Ordnance Survey Geoid Model 2002) với độ chính xác $\pm 2 \mathrm{~cm}$ (OSGM02: $\mathrm{A}$ New geoid model for the British Isles); Mô hình geoid của Australia AUSGeoid09 với độ chính xác $\pm 3 \mathrm{~cm}$ đang được định hướng cho việc xây dựng hệ quy chiếu địa tâm GDA2020 (The Geocentric Datum of Australia) (Australia is on the move GDA2020); Hệ quy chiếu tọa độ không gian của Cộng hòa Liên bang Đức ETRS89/DREF91/2016 được xây dựng dựa trên mô hình quasigeoid GCG2016 (Germany Combined Quasigeoid of 2016) với độ chính xác $\pm 2 \mathrm{~cm}$ (Quasigeoid of the Federal Respublic of Germany).

Vậy mô hình quasigeoid quốc gia thực chất là cái gì. Sở dĩ phải đặt ra câu hỏi này bởi vì ở Việt Nam không hiếm các công trình đã coi mặt quasigeoid toàn cầu là mặt khởi tính cho hệ độ cao, trong khi đó hệ độ cao chuẩn quốc gia Hải Phòng 1972 (HP72) lại dựa trên mặt quasigeoid cục bộ Hòn Dấu sát nhất với mặt biển trung bình nhiều năm tại trạm nghiệm triều Hòn Dấu. Mô hình quasigeoid quốc gia là mạng lưới (grid) các ô chuẩn hình vuông (cells) nằm trên mặt quasigeoid quốc gia với các dữ liệu của mỗi đỉnh của ô chuẩn hình vuông bao gồm các toạ độ trắc địa $\mathrm{B}, \mathrm{L}$ trong hệ quy chiếu toạ 
độ quốc gia và độ cao quasigeoid cục bộ $\zeta$ là độ cao của đỉnh so với mặt ellipsoid quy chiếu quốc gia (ví dụ ở Việt Nam là ellipsoid WGS84 quy chiếu quốc gia), thêm vào đó độ cao quasigeoid được xác định từ đỉnh theo phương vuông góc với mặt ellipsoid quy chiếu quốc gia. Trong khi đó mô hình quasigeoid toàn cầu là mạng lưới các ô chuẩn hình vuông nằm trên mặt quasigeoid toàn cầu với các dữ liệu của mỗi đỉnh của ô chuẩn hình vuông bao gồm các toạ độ trắc địa $\bar{B}, \bar{E}$ trong hệ quy chiếu toạ độ quốc tế (ví dụ WGS84) và độ cao quasigeoid toàn cầu $\bar{\zeta}$ là độ cao của đỉnh so với mặt ellipsoid quy chiếu toàn cầu (ví dụ ellipsoid WGS84 quốc tế), thêm vào đó độ cao quasigeoid toàn cầu được xác định từ đỉnh theo phương vuông góc với mặt ellipsoid quy chiếu toàn cầu.

Với giải thích nêu trên, đối với điểm $M$ trên mặt vật lý Trái đất thuộc lãnh thổ quốc gia, các điểm tương ứng với nó là điểm $\mathrm{M}_{1}$ nằm trên mặt quasigeoid cục bộ (quốc gia) và điểm $\mathrm{M}_{2}$ nằm trên mặt quasigeoid toàn cầu (xem hình 1). Trên hình 1: $\mathscr{K}^{\prime}$ 'là độ cao chuẩn của điểm $\mathrm{M}$ trong hệ độ cao quốc gia;

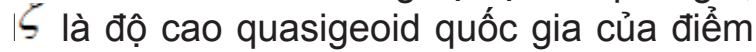
$\mathrm{M}$ và bằng đoạn $\mathrm{M}_{1} \mathrm{M}_{3}$; $\overline{\bar{\xi}}$ là độ cao quasi- geoid hỗn hợp của điểm $\mathrm{M}$ và bằng đoạn $\mathrm{M}_{1} \mathrm{Q}_{0} ; \bar{\zeta}$ là độ cao quasigeoid toàn cầu của điểm $\mathrm{M}$ và bằng đoạn $\mathrm{M}_{2} \mathrm{Q}_{0}$. Đại lượng bằng đoạn $M_{1} M_{2}$ là độ cao của mặt quasigeoid cục bộ Hòn Dấu so với mặt quasigeoid toàn cầu tương ứng với điểm $\mathrm{M}$ trên mặt vật lý Trái đất.

Sở dĩ $\overline{\bar{\zeta}}$ được gọi là độ cao quasigeoid hỗn hợp của điểm $\mathrm{M}$, bởi vì nó là độ cao của điểm $\mathrm{M}_{1}$ nằm trên mặt quasigeoid cục bộ (quốc gia) so với mặt ellipsoid quy chiếu quốc gia. Độ cao quasigeoid hỗn hợp không phải là độ cao quasigeoid quốc gia, tuy nhiên nó có vai trò rất quan trọng trong việc xây dựng mô hình quasigeoid quốc gia do hai lý do sau:

- Nó chính là độ cao quasigeoid GNSS/thủy chuẩn và được xác định từ công nghệ GNSS theo công thức:

$$
\overline{\bar{\zeta}}=\bar{H}-H^{\gamma},
$$

Ở đây $\bar{H}$ là độ cao trắc địa toàn cầu của điểm $M$ so với mặt ellipsoid quy chiếu toàn cầu và được xác định nhờ công nghê̂ GNSS, còn : $Z^{\prime}{ }^{\prime \prime}$ là độ cao chuẩn của điểm $\mathrm{M}$ trong hệ độ cao quốc gia;

- Nếu xác định được độ cao $D_{0}$ của mặt

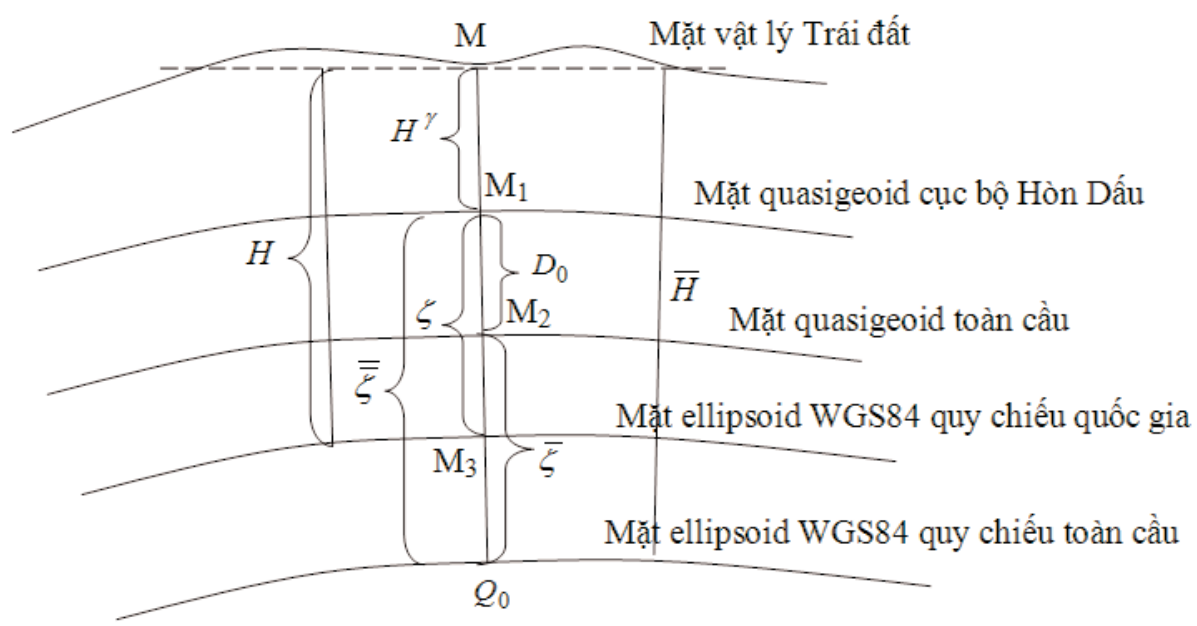

Hình 1: Các quan hệ giữa các mặt quasigeoid cục bộ, quasigeoid toàn cầu, ellipsoid quy chiếu quốc gia, ellipsoid quy chiếu toàn cầu 
quasigeoid cục bộ Hòn Dấu so với mặt quasigeoid toàn cầu, thì chúng ta hoàn toàn xác định được độ cao quasigeoid hỗn hợp theo công thức:

$$
\overline{\bar{\zeta}}^{*}=\bar{\zeta}+D_{0},
$$

ở đây $\bar{\zeta}$ là độ cao quasigeoid toàn cầu được xác định từ mô hình trọng trường Trái đất $E G M$ và đã được quy chuyển về ellipsoid WGS84 quy chiếu toàn cầu. Ở Việt Nam độ cao $D_{0}=0,890 m$ được xác định tại trạm nghiệm triều Hòn Dấu dựa trên hai nhóm dữ liệu độc lập:

+ Nhóm dữ liệu thứ nhất: Các độ cao trắc địa toàn cầu $\bar{H}$, độ cao quasigeoid toàn cầu $\bar{\xi}$ từ mô hình EGM2008 đã được quy đổi về ellipsoid WGS84 quy chiếu toàn cầu, độ cao chuẩn quốc gia " "k̆ của 89 điểm độ cao hạng | quốc gia và 75 điểm độ cao hạng || quốc gia;

+ Nhóm dữ liệu thứ hai: Mô hình mặt biển trung bình động lực DTU10MDT.

Các chứng minh lý thuyết và thực nghiệm xác nhận về sự không đổi của độ cao $D_{0}=0,890 m$ trên lãnh thổ Việt Nam và toàn cầu đã được trình bày trong nhiều tài liệu, ví dụ Hà Minh Hòa, và nnk, 2012; Hà Minh Hòa, 2016a; Hà Minh Hòa, Nguyễn Bá Thủy, Phan Trọng Trịnh và nnk, 2016b; Nguyễn Tuấn Anh, 2015; Lương Thanh Thạch, 2016.

Chính công thức (2) với $D_{0}=0,890 m$ đã được sử dụng để xây dựng mô hình quasigeoid hỗn hợp quốc gia VIGAC2014 (Hà Minh Hòa, Nguyễn Bá Thủy, Phan Trọng Trịnh và nnk, 2016b). Dựa trên 09 điểm cơ sở (là 09 điểm độ cao hạng I) của mô hình VIGAC2014 (xem chi tiết trong tài liệu Hà Minh Hòa, 2014b, ở trang 406) đã tiến hành thực nghiệm truyền độ cao nhà nước nhờ công nghệ GNSS và mô hình VIGAC2014 lên 30 điểm thuộc các mang lưới địa động lực Miền Bắc và vùng đồng bằng sông Cửu Long và ra hai đảo Côn Đảo và Phú Quốc với khoảng cách truyền cực đại đến $1500 \mathrm{~km}$. Các kết quả tính toán cho thấy tại mỗi điểm GNSS trong 09 giá trị độ cao chuẩn quốc gia được xác định, độ chênh giữa chúng không vượt quá $1,5 \mathrm{~cm}$ (xem tài liệu Hà Minh Hòa, Nguyễn Bá Thủy, Phan Trọng Trịnh và nnk, 2016b). Điều này cho thấy rằng mô hình quasigeoid toàn cầu EGM2008 nói chung và mô hình VIFAC2014 nói riêng cho độ chính xác rất cao của hiệu độ cao quasigeoid giữa hai điểm bất kỳ trên lãnh thổ Việt Nam.

Vậy vấn đề khoa học tiếp theo là làm thế nào để xây dựng được mô hình quasigeoid quốc gia dựa trên mô hình quasigeoid hỗn hợp VIGAC2014?. Để trả lời được câu hỏi này chúng ta phải xác định được quan hệ giữa mô hình VIGAC2014 với ellipsoid WGS84 quy chiếu quốc gia. Từ đây tất yếu phải giải quyết bài toán định vị ellipsoid WGS84 quy chiếu quốc tế cho sát nhất với mặt quasigeoid cục bộ Hòn Dấu. Bài toán này đã được giải quyết trong tài liệu $(\mathrm{Ha}$ Minh Hoa, 2017).

Khi định vị ellipsoid WGS84 quy chiếu quốc tế cho sát nhất với mặt quasigeoid cục bộ Hòn Dấu sao cho các trục tọa độ của ellipsoid WGS84 quy chiếu quốc tế và ellipsoid WGS84 quy chiếu quốc gia song song với nhau, chúng ta có quan hệ giữa độ cao trắc địa toàn cầu $\bar{H}$ và độ cao trắc địa quốc gia $H$ của điểm bất kỳ ở dạng sau:

$$
H=\bar{H}+A .\left(\begin{array}{l}
d X_{0} \\
d Y_{0} \\
d Z_{0}
\end{array}\right),
$$

ở đây ma trận hệ số $\mathrm{A}$ có dạng:

$$
A=(\cos \bar{B} \cdot \cos \bar{L} \quad \cos \bar{B} \cdot \sin \bar{L} \quad \sin \bar{B}),
$$

$\bar{B}, \bar{L}, \bar{H}$ là các tọa độ trắc địa của điểm tương ứng trên ellipsoid WGS-84 quy chiếu quốc tế nhận được từ các kết quả đo đạc GNSS và xử lý các dữ liệu đo GNSS trong ITRF; $d X_{0}, d Y_{0}, d Z_{0}$ là các tọa độ không gian của tâm ellipsoid WGS-84 quy chiếu toàn cầu so với tâm ellipsoid WGS-84 quy 
chiếu quốc gia.

Lưu ý độ cao quasigeoid quốc gia của điểm $\zeta=H-H^{\gamma}$ và công thức (1), từ (3) chúng ta có quan hệ giữa độ cao quasigeoid quốc gia $\zeta$ và độ cao quasigeoid hỗn hợp $\bar{\zeta}$ ở dạng sau:

$$
\zeta=\overline{\bar{\zeta}}+A \cdot\left(\begin{array}{l}
d X_{0} \\
d Y_{0} \\
d Z_{0}
\end{array}\right)
$$

Do độ cao quasigeoid hỗn hợp $\overline{\bar{\zeta}}$ hoàn toàn được xác định bằng công nghệ GNSS trên điểm có độ cao chuẩn quốc gia $\eta^{7}{ }^{7}$ nên khi giải hệ phương trình (4) trên $\mathrm{n}$ điểm GNSS/thủy chuẩn dưới điều kiện $\sum_{i=1}^{n} \xi^{2}=$ min, chúng ta sẽ xác định được các tham số $d X_{0}$, $d Y_{0}, d Z_{0}$ Từ đây lưu ý (4) chúng ta hoàn toàn chuyển đổi được mô hình quasigeoid hỗn hợp VIGAC2014 thành mô hình quasigeoid quốc gia khởi đầu theo công thức:

$$
\zeta^{*}=\bar{\zeta}^{*}+A \cdot\left(\begin{array}{l}
d X_{0} \\
d Y_{0} \\
d Z_{0}
\end{array}\right),
$$

Dựa trên164 điểm GPS/thủy chuẩn phủ trùm cả nước bao gồm 89 điểm độ cao hạng | và 75 điểm độ cao hạng II, thêm vào đó các kết quả đo GPS và xử lý các dữ liệu đo GPS trong ITRF bằng phần mềm Bernese đã được Cục Đo đạc và Bản đồ Việt Nam thực hiện trong giai đoạn 2009 - 2010, trong tài liệu liệu (Hà Minh Hoà, 2017) đã công bố các tham số chuyển đổi từ ITRF tương ứng với ellipsoid WGS84 quy chiếu quốc tế về hệ quy chiếu tọa độ không gian quốc gia khởi đầu VN2000-3D như sau:

$$
\begin{aligned}
& d X_{0}=204,511083 \mathrm{~m}, \\
& d Y_{0}=42,192468 \mathrm{~m}, \\
& d Z_{0}=111,417880 \mathrm{~m} . \\
& \text { Ex <giay> }=-0,011168229, \\
& \text { Ey <giay> }=0 ", 085600577, \\
& \text { Ez < giay> }=-0,, 400462723
\end{aligned}
$$

\section{$\mathrm{Dm}=0,000000000$}

Việc đánh giá độ chính xác của độ cao quasigeoid quốc gia từ hai dãy các giá trị độ cao quasigeoid độc lập: Dãy thứ nhất bao gồm 164 độ cao quasigeoid $\zeta$ được xác định từ công thức (4), dãy thứ hai bao gồm 164 độ cao quasigeoid $\zeta^{*}$ được xác định từ công thức (5) dựa trên mô hình VIGAC2014 cho thấy độ chính xác của độ cao quasigeoid quốc gia đạt ở mức

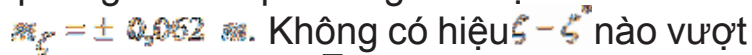
quá hạn sai $\pm 2,5 \cdot \sqrt{2} \cdot m_{\zeta}= \pm 0,219 m$, thêm vào đó 160 (chiếm 97,56 \%) các hiệu $\xi-\xi^{3}$ nhỏ hơn hạn sai $\pm 2,0 \cdot \sqrt{2} \cdot m_{\zeta}= \pm 0,175 \mathrm{~m}$. Điều này một lần nữa xác nhận sự tin cậy của mô hình VIGAC2014 được xác định theo công thức (2) với $D_{0}=0,890 \mathrm{~m}$. Mô hình quasigeoid quốc gia khởi đầu VIGAC2017 được xây dựng dựa trên công thức (5) và hệ quy chiếu toạ độ không gian quốc gia khởi đầu (VN2000-3D) liên kết với ITRF theo 07 tham số chuyển toạ độ nêu trên.

Mục đích của bài báo khoa học này là đánh giá độ chính xác của mô hình VIGAC2017 dựa trên 25 điểm GPS/thủy chuẩn không tham gia định vị ellipsoid và nghiên cứu sự thay đổi các toạ độ trắc địa $B, L$ giữa VN2000-3D và VN2000-2D.

\section{Giải quyết vấn đề}

Do các điểm độ cao hạng I, II quốc gia bị xê dịch nhiều do các yếu tố tự nhiên và nhân sinh, nên trong các đề tài (Hà Minh Hòa, và nnk, 2012; Hà Minh Hòa, Nguyễn Bá Thủy, Phan Trọng Trịnh và nnk, 2016b; Lương Thanh Thạch, 2016) đã tiến hành kiểm tra sự ổn định của các điểm độ cao hạng I, II theo các phương pháp khác nhau. Trong tổng số 230 điểm độ cao hạng I và 199 điểm độ cao hạng II được Cục Đo đạc và Bản đồ Việt Nam đo GPS và xử lý dữ liệu đo GPS trong ITRF tương ứng với ellipsoid WGS84 quy chiếu quốc tế đã xác định được 97 điểm độ cao hạng I và 75 điểm độ cao hạng II ổn định nhất. Để giải quyết định vị 
ellipsoid được đề cập ở trên đã sử dụng 89 điểm độ cao hạng I cùng với 75 điểm độ cao hạng II. 08 điểm độ cao hạng I còn lại được sử dụng để đánh giá độ chính xác của mô hình VIGAC2017. Ngoài ra trong khuôn khổ các đề tài nêu trên, Viện Khoa học Đo đạc và Bản đồ đã đo GPS trên điểm độ cao hạng I (BH-TH)112 và 16 điểm GPS được đo nối từ các điểm độ cao hạng I. Các kết quả tính chuyển các toạ độ trắc địa từ ellipsoid WGS84 quy chiếu quốc tế về hệ VN2000-3D tương ứng với ellipsoid WGS84 quy chiếu quốc gia và các kết quả tính toán độ cao chuẩn quốc gia $\hat{H}^{\gamma}$ của 25 điểm kiểm tra dựa trên mô hình quasigeoid quốc gia khởi đầu VIGAC2017 và so sánh với các độ cao chuẩn quốc gia敢 (các độ cao gốc quốc gia trong hệ độ cao HP72) (xem bảng 1).

Khi coi các giá trị độ cao chuẩn quốc gia (các độ cao gốc quốc gia trong hệ độ cao HP72) của các điểm độ cao hạng I kiểm tra là không có sai số, dựa trên công thức Gauss chúng ta có độ chính xác của độ cao chuẩn quốc gia được xác định nhờ hệ quy chiếu toạ độ không gian khởi đầu VN2000-3D và mô hình quasigeoid quốc gia khởi đầu VIGAC2017 như sau:

$$
m_{\hat{H}}^{\gamma}= \pm \sqrt{\frac{\sum_{i=1}^{25} d_{i}^{2}=0.148876}{25}}= \pm 0,077 \mathrm{~m} .
$$

Do 07 tham số chuyển toạ độ từ ITRF về VN2000-3D được trình bày ở mục 1 khác với 07 tham số chuyển toạ độ từ ITRF về VN2000-2D của Cục Đo đạc và Bản đồ Việt Nam [xem Hướng dẫn sử dụng các tham số tính chuyển từ Hệ toạ độ quốc tế WGS84 sang Hệ toạ độ quốc gia VN-2000 và ngược lại], nên các toạ độ trắc địa của điểm trắc địa được xác định trong VN2000-3D khác với các toạ độ trắc địa tương ứng trong VN2000-2D, nhưng rất nhỏ. Trong bài báo khoa học này đã trình bày các kết quả so sánh các toạ độ trắc địa giữa VN2000-3D và VN2000-2D đối với 10 điểm GNSS để minh hoạ (xem bảng 2).

Để đảm bảo sự không thay đổi các toạ độ trắc địa giữa VN2000-3D và VN2000-2D, đối với VN2000-3D các vĩ độ trắc địa $B$ được hiệu chỉnh bởi số cải chính

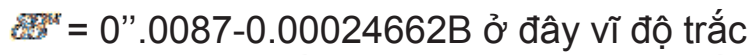
địa $B$ được quy về độ, còn các kinh độ trắc địa $L$ được hiệu chỉnh bởi số hiệu chỉnh ख्य $=0 " .002$.

\section{Bảng 1}

\begin{tabular}{|c|c|c|c|c|c|}
\hline STT & Tên điểm & $\begin{array}{l}\text { Các toạ độ trắc địa } \\
\text { quốc gia } B, L, H \text { trong } \\
\text { VN2000-3D }\end{array}$ & 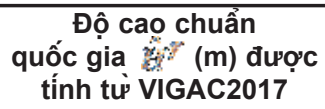 & $\begin{array}{c}\text { Độ cao chuẩn quốc } \\
\text { gia }{ }^{2}(\mathrm{~m}) \text { trong hệ độ } \\
\text { cao HP72 }\end{array}$ & 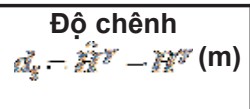 \\
\hline \multicolumn{6}{|c|}{ Các mốc độ cao hạng I được Cục Đo đạc và Bản đồ Việt Nam đo GPS trong giai đoạn 2009 - 2010} \\
\hline 1 & I(BT-PD)46 & $\begin{array}{c}113040.297 \\
1064540.092 \\
51.139 \mathrm{~m}\end{array}$ & 50.745 & 50.834 & -0.089 \\
\hline 2 & I(VL-HT)305 & $\begin{array}{c}10 \quad 0717.163 \\
1051713.823 \\
0.560 \mathrm{~m}\end{array}$ & 1.319 & 1.403 & -0.084 \\
\hline 3 & IBH-LS94-1 & $\begin{array}{c}220141.186 \\
1063745.846 \\
194.372 \mathrm{~m} \\
\end{array}$ & 195.272 & 195.146 & 0.126 \\
\hline 4 & $\mathrm{I}(\mathrm{HN}-\mathrm{VL}) 76$ & $\begin{array}{c}175619.670 \\
1062830.961 \\
12.676 \mathrm{~m} \\
\end{array}$ & 15.310 & 15.176 & 0.134 \\
\hline 5 & $\mathrm{I}(\mathrm{VL}-\mathrm{HT}) 113$ & $\begin{array}{c}135636.450 \\
1090436.947 \\
8.209 \mathrm{~m}\end{array}$ & 8.415 & 8.288 & 0.127 \\
\hline 6 & I(BT-NH)11-1 & $\begin{array}{c}124818.513 \\
1083219.267 \\
468.855 \mathrm{~m}\end{array}$ & 466.779 & 466.648 & 0.131 \\
\hline
\end{tabular}


Nghiên cứu

\begin{tabular}{|c|c|c|c|c|c|}
\hline 7 & I(DN-BT)18-1 & $\begin{array}{c}153343.638 \\
1074910.383 \\
112.327 \mathrm{~m}\end{array}$ & 112.429 & 112.514 & -0.085 \\
\hline 8 & IHP-MC9 & $\begin{array}{c}205848.423 \\
1065508.766 \\
6.558 \mathrm{~m}\end{array}$ & 8.512 & 8.624 & -0.112 \\
\hline \multicolumn{6}{|c|}{ Các mốc độ cao hạng I được Viện Khoa học Đo đạc và Bản đồ đo GPS trong các năm 2012 - 2013} \\
\hline 9 & QN01 & $\begin{array}{c}153506.413 \\
1082754.607 \\
1.967 \mathrm{~m}\end{array}$ & 3.468 & 3.475 & -0.007 \\
\hline 10 & QNG01 & $\begin{array}{c}145410.818 \\
1085442.684 \\
3.606 \mathrm{~m}\end{array}$ & 4.666 & 4.753 & -0.087 \\
\hline 11 & BP01 & $\begin{array}{c}112418.133 \\
1063640.250 \\
49.531 \mathrm{~m}\end{array}$ & 49.426 & 49.402 & 0.024 \\
\hline 12 & $22 \mathrm{~A} 1$ & $\begin{array}{c}20 \quad 0222.308 \\
1055032.487 \\
1.058 \mathrm{~m}\end{array}$ & 1.901 & 1.913 & -0.012 \\
\hline 13 & $38 \mathrm{~A} 1$ & $\begin{array}{c}191416.835 \\
1054147.602 \\
2.758 \mathrm{~m}\end{array}$ & 4.074 & 4.153 & -0.079 \\
\hline 4 & VL48 & $\begin{array}{c}184649.762 \\
1053843.945 \\
3.271 \mathrm{~m}\end{array}$ & 4.864 & 4.834 & 0.030 \\
\hline 15 & VL59 & $\begin{array}{c}182410.621 \\
1054920.297 \\
-0.118 \mathrm{~m}\end{array}$ & 1.752 & 1.752 & 0.0 \\
\hline 16 & HT84 & $\begin{array}{c}150435.941 \\
1084947.204 \\
3.347 \mathrm{~m}\end{array}$ & 4.560 & 4.496 & 0.064 \\
\hline 17 & НT94 & $\begin{array}{c}144432.031 \\
1090137.841 \\
8.319 \mathrm{~m}\end{array}$ & 9.353 & 9.241 & 0.112 \\
\hline 18 & HT106 & $\begin{array}{c}141629.068 \\
1090438.062 \\
12.138 \mathrm{~m}\end{array}$ & 12.562 & 12.534 & 0.028 \\
\hline 19 & HT121 & $\begin{array}{c}134548.672 \\
1090854.898 \\
8.757 \mathrm{~m}\end{array}$ & 8.730 & 8.765 & 0.035 \\
\hline 20 & HT127-4 & $\begin{array}{c}132302.209 \\
1091428.796 \\
3.231 \mathrm{~m}\end{array}$ & 2.773 & 2.760 & 0.012 \\
\hline 21 & HT141-3 & $\begin{array}{c}125217.776 \\
1092354.474 \\
151.460 \mathrm{~m}\end{array}$ & 150.542 & 150.479 & 0.063 \\
\hline 22 & HT159-1 & $\begin{array}{c}122211.178 \\
1091130.536 \\
11.035 \mathrm{~m}\end{array}$ & 9.565 & 9.602 & -0.037 \\
\hline 23 & HT173-3 & $\begin{array}{c}115803.888 \\
1091121.069 \\
4.5094 \mathrm{~m}\end{array}$ & 2.950 & 2.983 & -0.033 \\
\hline 24 & HT197 & $\begin{array}{c}111342.522 \\
1084248.132 \\
18.673 \mathrm{~m}\end{array}$ & 16.768 & 16.690 & 0.078 \\
\hline 25 & $\mathrm{I}(\mathrm{BH}-\mathrm{TH}) 112$ & $\begin{array}{c}204824.670 \\
10445 \quad 1.409 \\
976.807 \mathrm{~m}\end{array}$ & 974.866 & 974.851 & 0.015 \\
\hline
\end{tabular}


Bảng 2:

\begin{tabular}{|c|c|c|c|c|}
\hline STT & Tên điểm & $\begin{array}{l}\text { B, L, H trong } \\
\text { VN2000-3D }\end{array}$ & $\begin{array}{l}\text { B, L trong } \\
\text { VN2000-2D }\end{array}$ & $\begin{array}{l}\mathrm{dB} \\
\mathrm{dL}\end{array}$ \\
\hline \multicolumn{5}{|c|}{ Lưới địa động lực Miền Bắc (2013) } \\
\hline 1 & C052 & $\begin{array}{rll}2138 & 11.879 \\
104 & 47 & 07.206 \\
& 88.557\end{array}$ & $\begin{array}{c}213811.882 \\
1044707.209\end{array}$ & $\begin{array}{l}-0 " .003 \\
-0 " .003\end{array}$ \\
\hline 2 & $\mathrm{C} 022$ & $\begin{array}{c}210147.286 \\
1041835.056 \\
439.674\end{array}$ & $\begin{array}{c}210147.289 \\
1041835.058\end{array}$ & $\begin{array}{l}-0 " .003 \\
-0 " .002\end{array}$ \\
\hline 3 & C045 & $\begin{array}{c}210710.794 \\
1045847.932 \\
180.337\end{array}$ & $\begin{array}{c}210710.797 \\
1045847.934\end{array}$ & $\begin{array}{l}-0 " .003 \\
-0 " .002\end{array}$ \\
\hline 4 & C033 & $\begin{array}{c}213300.424 \\
1040201.514 \\
286.608\end{array}$ & $\begin{array}{c}213300.427 \\
1040201.517\end{array}$ & $\begin{array}{l}-0 " .003 \\
-0 " .002\end{array}$ \\
\hline 5 & C004 & $\begin{array}{c}215536.200 \\
1031410.998 \\
\quad 371.261\end{array}$ & $\begin{array}{c}215536.205 \\
1031411.000\end{array}$ & $\begin{array}{l}-0 " .005 \\
-0 " .002\end{array}$ \\
\hline \multicolumn{5}{|c|}{ Lưới địa động lực vùng đồng bằng Sông Cửu Long (2013) } \\
\hline 6 & A004 & $\begin{array}{c}100543.890 \\
1052533.718 \\
9.493\end{array}$ & $\begin{array}{cc}10 & 0543.897 \\
105 & 2533.719\end{array}$ & $\begin{array}{l}-0 " .007 \\
-0 " .001\end{array}$ \\
\hline 7 & A001 & $\begin{array}{c}101325.201 \\
1055851.792 \\
2.618\end{array}$ & $\begin{array}{r}101325.207 \\
1055851.792\end{array}$ & $\begin{array}{c}-0 " .006 \\
0 " .0\end{array}$ \\
\hline 8 & A011 & $\begin{aligned} 91416.460 \\
1052715.474 \\
2.4732\end{aligned}$ & $\begin{array}{r}91416.466 \\
1052715.475\end{array}$ & $\begin{array}{l}-0 " .006 \\
-0 " .001\end{array}$ \\
\hline 9 & A012 & $\begin{array}{c}85127.628 \\
105019.617 \\
2.216\end{array}$ & $\begin{array}{r}85127.635 \\
105 \quad 019.619\end{array}$ & $\begin{array}{l}-0 " .007 \\
-0 " .002\end{array}$ \\
\hline 10 & A009 & $\begin{array}{c}836 \quad 05.311 \\
1044518.124 \\
2.423\end{array}$ & $\begin{array}{r}83605.317 \\
1044518.126\end{array}$ & $\begin{array}{l}-0 " .006 \\
-0 " .002\end{array}$ \\
\hline
\end{tabular}

\section{Kết luận}

Các kết quả kiểm tra độ chính xác của mô hình quasigeoid quốc gia khởi đầu VIGAC2017 trong hệ quy chiếu toạ độ không gian quốc gia khởi đầu VN2000-3D cho thấy mô hình này có độ chính xác khá cao và đảm bảo việc xác định độ cao chuẩn quốc gia hạng II nhờ công nghệ GNSS. Việc nâng cao tiếp theo độ chính xác của mô hình quasigeoid quốc gia sẽ được thực hiện nhờ sử dụng các dữ liệu đo trọng lực chi tiết trong tương lai. $O$

\section{Tài liệu tham khảo}

[1]. Augath, W., Ihde, J., 2002. Definition and realization of Vertical Reference System - the European Solution EVRS/EVRF2000. FIG XXII International Congress, Washington D.C., April 19-26 2002.

[2]. Australia is on the move GDA2020.

https://esriaustraliatechblog.wordpress.c om/2016/08/12/australia-is-on-the-movegda2020/

[3]. Hà Minh Hòa, và nnk, 2012. Nghiên 
cứu cơ sở khoa học của việc hoàn thiện hệ độ cao gắn liền với việc xây dựng hệ tọa độ động lực quốc gia. Đề tài khoa học và công nghệ cấp Bộ Tài nguyên và Môi trường giai đoạn 2010 - 2012. Hà Nội - 2012.

[4]. Hà Minh Hòa, 2014a. Phương pháp xử lý toán học các mạng lưới trắc địa quốc gia. Nhà Xuất bản Khoa học và Kỹ thuật, 244 trg., Hà Nội - 2014.

[5]. Hà Minh Hòa, 2014b. Lý thuyết và thực tiễn của Trọng lực trắc địa. Nhà Xuất bản Khoa học và Kỹ thuật, 592 trg., Hà Nội - 2014.

[6]. Hà Minh Hòa, 2016a. Nghiên cứu sự thay đổi độ cao giữa mặt quasigeoid cục bộ Hòn Dấu và mặt quasigeoid toàn cầu trên phạm vi toàn cầu. Tạp chí Khoa học Đo đạc và Bản đồ, số 28, tháng 06/2016, trg. 1 - 7.

[7]. Hà Minh Hòa, Nguyễn Bá Thủy, Phan Trọng Trịnh, Nguyễn Nguyên Cương, Nguyễn Phi Sơn, Nguyễn Thị Thanh Hương, và nnk, 2016b. "Nghiên cứu đánh giá các mặt chuẩn mực nước biển (mặt " 0 " độ sâu, trung bình và cao nhất) theo các phương pháp trắc địa, hải văn và kiến tạo hiện đại phục vụ xây dựng các công trình và quy hoạch đới bờ Việt Nam trong xu thế biến đổi khí hậu". Đề tài khoa học và phát triển công nghệ mã số KC.09.19/11 -15 giai đoạn 2012 - 2015 thuộc Chương trình KH\&CN trọng điểm cấp Nhà nước KC09/11-15 "Nghiên cứu khoa học và công nghệ phục vụ quản lý biển, hải đảo và phát triển kinh tế biến" giai đoạn 2011 - 2015. Bộ
Khoa học và Công nghệ, Hà Nội - 2016.

[8]. Ha Minh Hoa, 2017. Construction of inital national quasigeoid model VIGAC2017 - First step to national spatial reference system in Vietnam. Vietnam journal of Earth Sciences, 39 (2): 155 - 166, Vietnam Academy of Science and Technology, http://www.vjs.ac.vn/index.php/jse.

[9]. Hướng dẫn sử dụng các tham số tính chuyển từ Hệ toạ độ quốc tế WGS84 sang Hệ toạ độ quốc gia VN-2000 và ngược lại. Công văn số 1123/ĐĐBĐ-CNTĐ ngày 26/10/2007 của Cục trưởng Cục Đo đạc và Bản đồ Việt Nam

[10]. Lương Thanh Thạch, 2016. Đánh giá độ chính xác của mô hình quasigeoid quốc gia khởi đầu VIGAC2014 dựa trên cơ sở dữ liệu của 75 điểm độ cao hạng II. Tạp chí Khoa học Đo đạc và Bản đồ số 30 , tháng 12/2016, trg. $17-28$.

[11]. Nguyễn Tuấn Anh, 2015. Nghiên cứu chi tiết độ cao của mặt geoid cục bộ Hòn Dấu so với mặt geoid toàn cầu trên lãnh thổ Việt Nam. Tạp chí Khoa học Đo đạc và Bản đồ, No25, 09/2015, pp. 33 - 38.

[12]. Quasigeoid of the Federal Respublic of Germany. Geodatenzentrum, https://upd.geodatenzentrum.de/docpdf/qua sigeoid_eng.pdf

[13]. OSGM02: A New geoid model for the British Isles. http://cct.gfy.ku.dk/publ_cct/cct1777.pdf O

\section{Summary}

\section{Accuracy estimation of initial national quasigeoid model VIGAC2017}

Ha Minh Hoa, Vietnam Institute of Geodesy and Cartography

\section{Luong Thanh Thach, Hanoi University of National Resources and Environment}

This scientific article presents results of construction of initial national quasigeoid model VIGAC2017 with accuracy at level $\pm 6,2 \mathrm{~cm}$ in a initial national spatial reference system VN2000 - 3D. Estimation results show that the initial national quasigeoid model VIGAC2017 has high reliability and accuracy. $O$ 